Ann. Biol. anim. Bioch. Biophys., 1979, 19 (6), 1689-1699.

\title{
Dosage des œstrogènes libres et conjugués dans le plasma périphérique de vache cyclique
}

\author{
par A. LOPEZ-SEBASTIAN*, J. SAUMANDE \\ Station de Physiologie de la Reproduction, I. N. R. A., \\ Nouzilly 37380 Monnaie, France.
}

Summary. Assay of free and conjugated oestrogens in peripheral plasma of cyclic cows.

Conjugated oestrone $\left(E_{1}\right)$, oestradiol- $17 \beta\left(E_{2} \beta\right)$, and oestradiol-17 $\alpha\left(E_{2} \alpha\right)$ were measured in the same plasma sample of cyclic cows. After enzymatic hydrolysis oestrogens were extracted with diethyl ether and an initial chromatography on a micro-colum of Sephadex LH 20 allowed the separation of $E_{1}$ from both $E_{2}$. As it is very difficult to separate the 2 epimers of $E_{2}, E_{2} \beta$ was specifically oxidized to $E_{1}$ so that following a second chromatography, the $E_{2}$ fraction contained only $E_{2} \alpha$. Thereafter, the 3 oestrogens were quantified by radioimmunoassay. The method was validated for bovine plasma. As free oestrogens were not separated from the conjugated oestrogens prior to hydrolysis total $E_{1}, E_{2} \beta$ and $E_{2} \alpha\left(T E_{1}\right.$, $\left.\mathrm{TE}_{2} \beta, \mathrm{TE}_{2} \alpha\right)$ were assayed.

$T E_{1}, T_{2} \beta$ and $T E_{2} \alpha$ during the bovine cycle were at a higher level than the corresponding non conjugated estrogens. A preovulatory increase was evident only for $\mathrm{TE}_{2} \beta$ but neither its duration nor its relative increase were larger than that of $E_{2} \beta$.

Les variations des concentrations d'œstrogènes dans le sang veineux ovarien ou périphérique ou dans l'urine ont depuis longtemps été mises en relation avec l'activité folliculaire de l'ovaire. Dans l'espèce bovine, l'étude de l'évolution des concentrations d'œstradiol-17 $\beta\left(E_{2} \beta\right)$ dans le sang périphérique a permis de montrer que ce paramètre pouvait être un excellent reflet qualitatif et quantitatif de la"croissance folliculaire, aussi bien au cours du cycle sexuel (Henricks, Dickey et Hill, 1971 ; Glencross et al., 1973 ; Dobson et Dean, 1974 ; Lemon et Saumande, 1974 ; Chenault et al., 1975 ; Ectors et al., 1975 ; Peterson et al., 1975 ; Thibier et Saumande, 1975) que chez l'adulte ou l'impubère qui ont reçu des traitements de stimulation (Lemon et Saumande, 1972 ; Henricks et al., 1973 ; Saumande et Testart, 1974 ; Saumande et Pelletier, 1975 ; Testart et al., 1977 ; Saumande, 1978). Cependant, une telle étude se heurte à 2 difficultés qui en limitent l'utilisation :

- les concentrations $d^{\prime} E_{2} \beta$ à mesurer sont faibles : la plupart du temps, inférieures à $20 \mathrm{pg} / \mathrm{ml}$;

- elles varient rapidement, ce qui oblige, pour bien suivre un phénomène, à réaliser

\footnotetext{
* Adresse permanente : I. N. I. A.-CRIDA 06, Adva Puerta de Hierre S/N Madrid-3, Espagne.

Les abréviations suivantes ont été utilisées : $E_{1}$ : œstrone, $E_{2} \beta$ : œstradiol-17 $\beta, E_{2} \alpha$ : œstradiol-17 $\alpha$
} 
des prélèvements fréquents, toutes les $2 \mathrm{~h}$ en général (Lemon ef Saumande, 1974; Chenault et al., 1975 ; Peterson ef al., 1975 ; Thibier ef Saumande, 1975).

Dans le sang périphérique de la femme, il a été montré que les concentrations d'œstrone sulfate $\left(E_{1} S\right)$ sont respectivement 2 fois et 6 fois supérieures à celles d' $E_{2} \beta$ ef d' $E_{1}$ non conjugués et, surtout, que leurs variations relatives au cours du cycle sont plus importantes : le pic préovulatoire représente 11 fois le niveau de base pour $E_{1} S$ ef seulement 6 fois pour $E_{2} \beta$ (Brown et Smyth, 1971 ; Loriaux, Ruder et Lipsett, 1971 ; Hawkins ef Oakey, 1974). De plus, il a été observé que la demi-vie des stéroïdes sulfates est beaucoup plus longue que celle des stéroïdes non conjugués ( 5 à $9 \mathrm{~h}$ contre moins de $20 \mathrm{mn}$ ) (Longcope, 1972 ; Ruder, Loriaux et Lipsett, 1972 ; Dessypris, 1975). La mesure des œstrogènes conjugués pourrait donc être la solution non seulement des problèmes quantitatifs, mais aussi celui des variations rapides.

Des résultats préliminaires (Lemon et Saumande, 1974), acquis avec un anticorps non spécifique dirigé contre $E_{1}$ principalement, ont montré effectivement dans le plasma de vache cyclique la présence de quantités importantes d'œstrogènes conjugués, mais pas de variations nettes. C'est pour cela que nous avons entrepris une étude des variations des concentrations de $E_{1}, E_{2} \beta$ et $E_{2} \alpha$ totaux (non conjugués et conjugués après hydrolyse des conjugués ; ces trois œstrogènes ont été étudiés, car on sait qu'ils sont présents dans le plasma de bovin au cours du cycle (Dobson ef Dean, 1974). Nous rapportons, ici, les résultats concernant la mise au point de la technique et les premières données concernant les concentrations et l'évolution des trois œstrogènes au cours du cycle œstral.

\section{Matériel et méthodes.}

\section{Animaux.}

Les prélèvements sanguins ont été réalisés sur 3 vaches FPN cycliques une fois par jour pendant 1,5 cycle (866), 1 cycle (856) ou 8 jours centrés autour des chaleurs (854). Après centrifugation, le plasma est recueilli et congelé jusqu'au moment du dosage.

La détection d'œstrus est réalisée à l'aide d'un taureau vasectomisé qui esł mis en présence des animaux deux fois par jour. Le premier jour où la femelle accepte le chevauchement est défini comme $\mathrm{J}_{0}$.

\section{Matériel.}

Les stéroïdes de référence ont été obtenus chez Stéraloïds et utilisés sans purification préalable. Les œstrogènes marqués ont été fournis par Amersham Radiochemical Center (Amersham) : $(2,4,6,7,3 \mathrm{H})$ œstrone 85-105 Ci/mmol, $(2,4,6,7,3 \mathrm{H}) œ s t r a-$ diol-17 $\beta$ 85-105 Ci/mmol (2, 4 (N)-3H) œstradiol-17 $\alpha 43 \mathrm{Ci} / \mathrm{mmol}$.

L’hydrolyse des œstrogènes conjugués a été réalisée par voie enzymatique avec une préparation d'Hélix Pomatia titrant 100000 unités de $\beta$-glucuronidase ef 1000000 unités sulfatase $/ \mathrm{ml}$ (Industrie Biologique Française). Cette préparation est utilisée en solution à 5 p. 100 dans du tampon acétate $1 \mathrm{M} \mathrm{pH} \mathrm{4,8;0,5} \mathrm{ml} \mathrm{de} \mathrm{cette}$ solution ajouté à $5 \mathrm{ml}$ de plasma est suffisant pour hydrolyser plus de 90 p. 100 des œstrogènes conjugués (Saumande, résultat non publié). 
La séparation des épimers de $E_{2}$ étant très difficile, nous avons tourné la difficulté en oxydant spécifiquement la fonction $17 \beta$-hydroxyle et séparant ensuite $E_{1}$ (primitivement $E_{2} \beta$ ) et $E_{2} \alpha$. La spécificité de l'oxydation de la fonction $17 \beta$-ol dépend des condi-

\section{TABLEAU 1}

Oxydation enzymatique de l'oestradiol-17 $\beta$ et de l'oestradiol-17 $\alpha$ dans différentes conditions de durée d'incubotion et de concentrations d'enzyme (HSD) et de cofacteur (NAD)

a) Effef de la durée d'incubotion :

\begin{tabular}{|c|c|c|c|}
\hline Temps (mn) & 120 & 60 & 30 \\
\hline p. 100 de $E_{2} \beta$ oxydé........ & $\begin{array}{l}91,0 \\
92,0 \\
93,0\end{array}$ & $\begin{array}{l}94,3 \\
91,2 \\
93,0\end{array}$ & $\begin{array}{l}93,8 \\
94,7 \\
93,2\end{array}$ \\
\hline p. 100 de $E_{2} \alpha$ oxydé $\ldots \ldots \ldots$ & $\begin{array}{l}69,0 \\
71,0 \\
70,3\end{array}$ & $\begin{array}{l}52,6 \\
53,4 \\
53,0\end{array}$ & $\begin{array}{l}41,2 \\
46,6 \\
45,0\end{array}$ \\
\hline
\end{tabular}

Quantité de HSD : 1*; Quantité de NAD : 1*.

b) Effet de la concentration de NAD :

\begin{tabular}{|c|c|c|c|c|}
\hline Concentration NAD & 1 & $1 / 2$ & $1 / 10$ & $1 / 20$ \\
\hline p. 100 de $E_{2} \beta$ oxydé........ & $\begin{array}{l}93,8 \\
94,7 \\
93,2\end{array}$ & $\begin{array}{l}93,5 \\
95,0 \\
94,8\end{array}$ & $\begin{array}{l}93,8 \\
94,0 \\
94,5\end{array}$ & $\begin{array}{l}93,6 \\
94,2 \\
94,5\end{array}$ \\
\hline p. 100 de $E_{2} \alpha$ oxydé $\ldots \ldots \ldots$ & $\begin{array}{l}41,2 \\
46,6 \\
45,0\end{array}$ & $\begin{array}{l}34,5 \\
34,6 \\
33,0\end{array}$ & $\begin{array}{l}15,4 \\
16,0 \\
15,3\end{array}$ & $\begin{array}{l}11,3 \\
11,2 \\
11,6\end{array}$ \\
\hline
\end{tabular}

Durée d'incubation : $30 \mathrm{mn}$; Concentration HSD : 1.

c) Effet de la concentration de HSD :

\begin{tabular}{crrrr}
\hline Concentration HSD & 1 & $1 / 2$ & $1 / 5$ & $1 / 10$ \\
\hline & & & & \\
p. 100 de $E_{2} \beta$ oxydé. ......... & 93,6 & 94,6 & 95,0 & 94,2 \\
& 94,2 & 94,0 & 96,0 & 96,0 \\
p. 100 de $E_{2} \alpha$ oxydé $\ldots \ldots . .$. & 94,5 & 95,3 & 94,5 & 96,2 \\
& 11,3 & 9,2 & 4,4 & 3,1 \\
& 11,2 & 8,3 & 4,7 & 3,2 \\
& 11,6 & 8,1 & 4,6 & 3,2 \\
\hline
\end{tabular}

Durée d'incubation : $30 \mathrm{mn}$; Concentration de NAD : 1/20.

* La concentration 1 correspond à $2,5 \mathrm{mg}$ de HSD et 2,04 mg de NAD par tube. La température d'incubation est toujours $37^{\circ} \mathrm{C}$. 
tions de réaction. Pour choisir celles qui sont les plus favorables, nous avons étudié séparément pour $E_{2} \beta$ et $E_{2} \alpha$ l'influence de 3 paramètres : durée de l'incubation, concentration de l'enzyme, concentration du cofacteur. Pour cela, à la fin de la réaction, la phase aqueuse est extraite par $10 \mathrm{ml}$ d'éther. L'extrait est séché, puis chromatographié sur une microcolonne de Séphadex LH 20 identique à celle décrite plus loin ; les fractions $E_{1}$ et $E_{2}$ sont récupérées et le pourcentage d'oxydation déterminé en comparant la radioactivité récupérée. A la suite des résultats obtenus dans les différents essais (tabl. 1), pour les dosages, nous avons employé par tube $0,1 \mathrm{mg}$ de $\beta-\mathrm{NAD}$ (Sigma) et $0,25 \mathrm{mg}$ d'hydroxy-steroids deshydrogénase (préparation enzymatique de Pseudomonas testosteroni, Sigma en solution dans $1,1 \mathrm{ml}$ de tampon carbonate $1 \mathrm{M} \mathrm{pH} \mathrm{9,1;}$ les tubes sont laissés $30 \mathrm{mn}$ à $37^{\circ} \mathrm{C}$. Dans ces conditions de réaction, $96 \mathrm{p} .100 \mathrm{de}$ $E_{2} \beta$ sont oxydés et seulement 3 p. 100 de $E_{2} \alpha$.

Oestrone et œstradiol sont séparés sur des colonnes de Séphadex LH $20(6 \mathrm{~cm} \times 0,4)$ préparées et utilisées avec un mélange benzène/éthanol : 85/15. Dans ces conditions, les 2 épimers de l'œstradiol ne sont pas séparés. La séparation n'est pas affectée par les très nombreux lipides présents dans l'extrait (fig. 1).

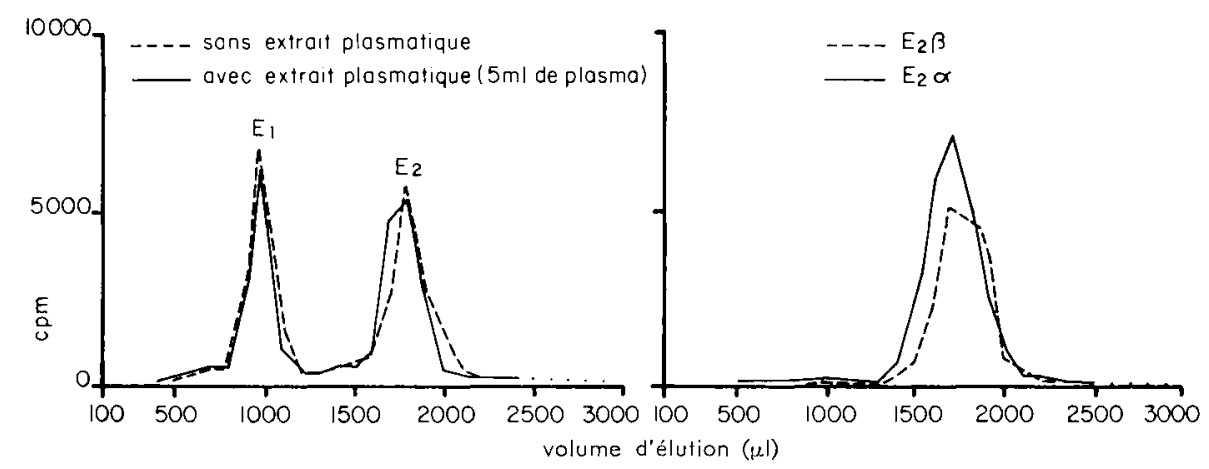

FIG. 1. - Séparation de $E_{1}$ ef $E_{2}$ par chromatographie sur une microcolonne de Sephadex LH $20: a$ ) Effet des impuretés présentes dans les échantillons plasmatiques. b) Elution des deux épimers de l'oestradiol.

Pour la partie radioimmunologique, le tampon utilisé est du tampon phosphate $1 \mathrm{M}, \mathrm{pH} 7$ qui contient $9 \mathrm{~g}$ de $\mathrm{NaCl}, 1 \mathrm{~g}$ d'azoture de $\mathrm{Na}$ et $1 \mathrm{~g}$ de gélatine par liłre.

Anticorps et technique de séparation des fractions libres ef liées, utilisés pour le dosage de $E_{2} \beta$ ont déjà été décrits (Thibier et Saumande, 1975 ; Testart et al., 1977). $E_{1}$ est mesuré avec un anticorps obtenu chez la brebis contre $E_{1}-6 \mathrm{CMO} \mathrm{BSA}^{*}$, $E_{2} \alpha$ avec un anticorps non spécifique obtenu aussi chez la brebis el dirigé contre $E_{1}-17-C M O$ qui présente une forte réaction croisée avec $\mathrm{E}_{2} \alpha(44 \mathrm{p}$. 100). Les 2 anticorps sont employés en dilution finale de $1 / 3000$ et $1 / 180000$.

Dans le dosage de $E_{1}$ et $E_{2} \alpha$ les fractions libres et liées sont séparées par la méthode du charbon-Dextran : $1 \mathrm{ml}$ d'une solution dans le tampon phosphate de charbon actif

* Nous remercions le Dr J. Adeline (FRH) pour le don de ses antisérums,. 
(Norit A, Nutritional Biochemicals Corporation) et de Dextran T70 (Pharmacia) respectivement $62,5 \mathrm{mg}$ et $2,5 \mathrm{mg} / 100 \mathrm{ml}$ sont ajoutés ; les tubes sont agités et, après $10 \mathrm{mn}$ de contact, centrifugés.

Pour mesurer la radioactivité liée, $0,8 \mathrm{ml}$ de surnageant est prélevé et transféré dans des fioles dans lesquelles on ajoute $10 \mathrm{ml}$ de liquide scintillant $(4 \mathrm{~g} P P O, 0,04 \mathrm{~g}$ POPOP par litre de toluène). Après agitation, la radioactivité est mesurée dans un compteur à scintillation liquide.

Méthodes de dosage.

- Préparation des échantillons.

Dans des tubes en verre, sont ajoutés successivement le plasma ( 2 à $5 \mathrm{ml})$, les œstrogènes radioactifs pour mesurer la récupération (environ $1000 \mathrm{cpm}$ de chaque) et la solution d'Helix Pomatia. Les tubes sont bouchés, agités au Vortex et laissés une nuit à $37^{\circ} \mathrm{C}$.

Comme les stéroïdes conjugués et non conjugués ne sont pas séparés avant hydrolyse, pour chacun des œstrogènes étudiés, il sera donc dosé la quantité totale présente dans le plasma; en conséquence, nous nous servirons par la suite des abréviations $T E_{1}, T E_{2} \beta$ ef $T E_{2} \alpha$ pour exprimer les résultats.

Les stéroïdes sont extraits avec de l'éther éthylique redistillée avant usage en utilisant 6 volumes d'éther pour un de solution aqueuse. Après agitation, la phase organique est récupérée et évaporée sous azote dans un bain-marie à $50^{\circ} \mathrm{C}$.

L'extrait sec est transféré sur une colonne de Séphadex LH 20 qui permet de séparer $E_{1}$ de la fraction $E_{2}$. Cette dernière est partagée en 2 : une moitié sert au dosage de $E_{2} \beta^{*}$, l'autre est soumise à l'oxydation de la fonction 17 $\beta-$ ol. Après incubation, les stéroïdes sont extraits par l'éther ; la fraction $E_{2}$ recueillie après une $2^{\mathrm{e}}$ chromatographie ne contient plus que $\mathrm{E}_{2} \alpha$.

- Dosage radioimmunologique.

Pour $E_{2} \beta$ la méthode est la même que celle publiée précédemment (Thibier et Saumande, 1975 ; Testart et al., 1977). Pour $E_{1}$ et $E_{2} \alpha$ l'éluat est séché puis repris dans $300 \mu$ l de tampon : $50 \mu \mathrm{l}$ permettront de mesurer la récupération, et sur 2 aliquots de $100 \mu \mathrm{l}$ sera réalisé le dosage. Pour cela, sont ajoutés dans $100 \mu \mathrm{l}$ de tampon environ $5000 \mathrm{cpm}$ d'œstrogène radioactif et, dans 100 autres $\mu$ l, l'anticorps; après une nuit d'incubation à $4^{\circ} \mathrm{C}$ ou $2 \mathrm{~h}$ à la température du laboratoire ef $1 \mathrm{~h}$ à $4{ }^{\circ} \mathrm{C}$, la fraction liée est ensuite séparée et comptée.

Les calculs sont réalisés avec une machine programmable Hewlett Packard après transformation dans le système Logit/Log.

\section{Résultats.}

Récupération. - Avant de procéder au dosage radioimmunologique, sont en solution dans le tampon 79, 40 et 33 p. 100 de l'échantillon de départ, respectivement

* Nous avons dû procéder ainsi car après la 2 e chromatographie, le dosage de $E_{1}$ (précédemment $\left.E_{2} \beta\right)$ n'est pas possible et ce, pour des raisons indéterminées. 
pour $E_{1}, E_{2} \beta$ et $E_{2} \alpha$. Après la 1 re chromatographie, la fraction $E_{2}$ est séparée en 2 afin de pouvoir doser $E_{2} \beta$; ceci explique la faible récupération des 2 cstradiols.

Blancs. - Malgré la purification de l'éther par distillation et de la préparation d'Hélix Pomatia par traitement au charbon actif, ces 2 produits sont responsables de blancs non nuls (respectivement 4 ef 12,5 pg en équivalent $E_{1}$ ). Nous avons donc ajouté dans la gamme ces substances interférentes en quantités équivalentes à celles retrouvées dans les échantillons. Le blanc est alors inférieur à la limite de détection des gammes pour les 3 œstrogènes.

Sensibilité. - Les limites de détection $\left({ }^{1}\right)$ des courbes standards sont 1 pg, 6,25 pg ef $12,5 \mathrm{pg}$ respectivement pour $\mathrm{E}_{2} \beta, \mathrm{E}_{2} \alpha$ et $\mathrm{E}_{1}$. La sensibilité des courbes standards a été calculée pour chaque point car la variance n'est pas homogène ; les résultats sont donnés dans le tableau 2.

TABLEAU 2

Sensibilité des courbes standards

\begin{tabular}{cccc}
\hline \multirow{2}{*}{$\begin{array}{c}\text { Point de la gamme }(\mathrm{pg}) \\
\left(\mathrm{G}_{1}\right)\end{array}$} & \multicolumn{3}{c}{$\begin{array}{c}\text { Quantité nécessaire pour avoir } \\
\text { un effet sig nificatif }\end{array}$} \\
\cline { 2 - 4 } & $\left.\mathrm{E}_{1}-\mathrm{G}_{1}\right)(\mathrm{pg})$ & $\mathrm{E}_{2} \beta$ & \\
\hline & & & $\mathrm{E}_{2} \alpha$ \\
0,78 & 21,15 & 1,32 & 2,58 \\
1,56 & 7,27 & 1,90 & 2,33 \\
3,12 & 7,23 & 1,46 & 2,18 \\
6,25 & 6,99 & 1,86 & 2,57 \\
12,5 & 1,42 & $* *$ & 1,48 \\
25 & 1,37 & 1,27 & 1,12 \\
50 & 1,28 & 1,09 & 1,17 \\
100 & 1,10 & 1,27 & 1,24 \\
200 & 1,12 & 1,31 & 1,26 \\
& & & \\
\hline
\end{tabular}

* Pour chaque point de gamme $\left(G_{1}\right)$ nous avons calculé $G_{2}$ qui vérifie l'inégalité

$$
\left|\left(G_{2}-G_{1}\right)\right|>\frac{2 \text { ts } \sqrt{2}}{u \sqrt{n}}
$$

où $\dagger=$ valeur de la table de Student, $s=$ déviation standard, $u=$ pente de la courbe standard, $\mathbf{n}=$ nombre de mesure par point.

** Ce point n'était sur aucune des droites.

Compte tenu de la récupération et du volume de tampon sur lequel la mesure est réalisée, la sensibilité du dosage est approximativement $5 \mathrm{pg}, 9 \mathrm{pg}$ et $11 \mathrm{pg}$ pour $E_{1}, E_{2} \beta$ et $E_{2} \alpha$.

Spécificité. - Pour $E_{1}$ ef $E_{2} \beta$, la spécificité des anticorps suffirait, à elle seule, à conférer au dosage une spécificité suffisante. Pour $E_{2} \alpha$, les œstrogènes susceptibles

(1) Plus petite quantité pour laquelle on observe une diminution significative $(P<0,05)$ par rapport au zéro de la radioactivité liée. 
d'interférer principalement $E_{1}$ et $E_{2} \beta$ sont éliminés lors de la préparation de l'échantillon (oxydation enzymatique et chromatographie).

Exactifude et précision. - Chacun des 3 œstrogènes éfudiés a été dosé dans 4 échantillons de 2, 4 et $6 \mathrm{ml}$ de plasma d'un même pool. Les résultats sont présentés dans le tableau 3. A sensibilité équivalente, la précision est d'autant meilleure que le dosage radioimmunologique est réalisé sur une fraction importante de l'échantillon de départ ; ceci explique qu'elle soit la meilleure pour $E_{1}$ puis $E_{2} \beta$ et $E_{2} \alpha$.

TABLEAU 3

Mesure de $\mathrm{TE}_{1}, \mathrm{TE}_{2} \beta$ ef $\mathrm{TE}_{2} \alpha$ dans différents volumes de plasma

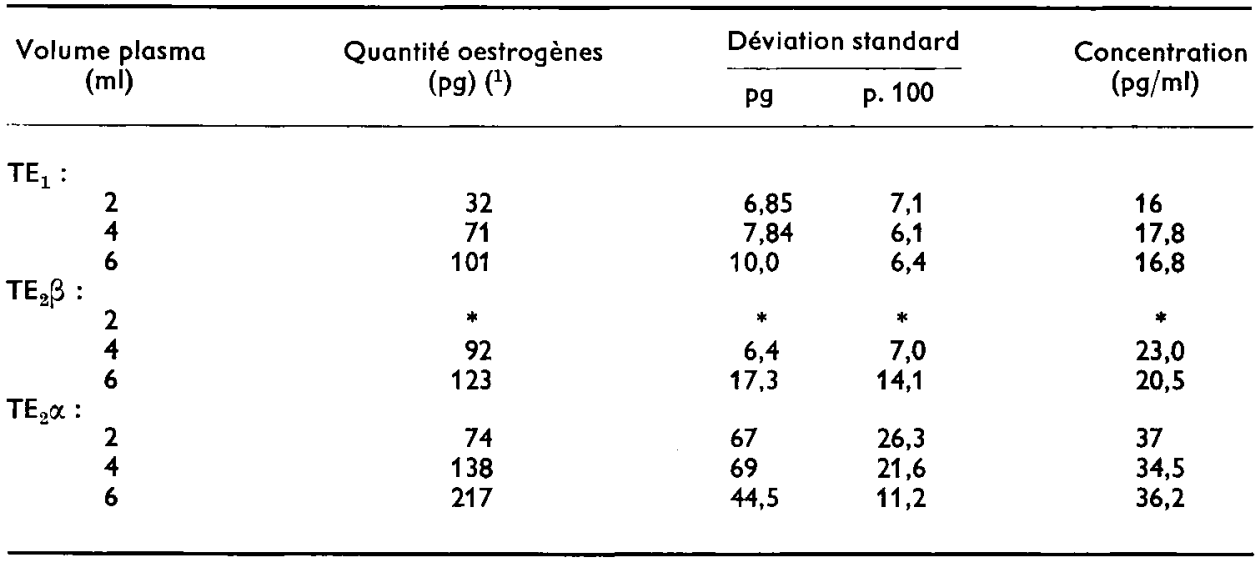

(1) Moyenne de 4 échantillons.

* Les quantités de départ ne permettaient pas un dosage compte tenu de la sensibilité du dosage.

Niveau de $\mathrm{TE}_{1}, \mathrm{TE}_{2} \beta$ ef $\mathrm{TE}_{2} \alpha$ au cours du cycle. - Les résultats de dosage sont donnés dans la figure 2 et le tableau 4. Du point de vue quantitatif, les concentrations les plus élevées sont mesurées pour $\mathrm{TE}_{2} \alpha(15$ à $70 \mathrm{pg} / \mathrm{ml})$ puis pour $\mathrm{TE}_{1}(15$ à $34 \mathrm{pg} / \mathrm{ml})$,

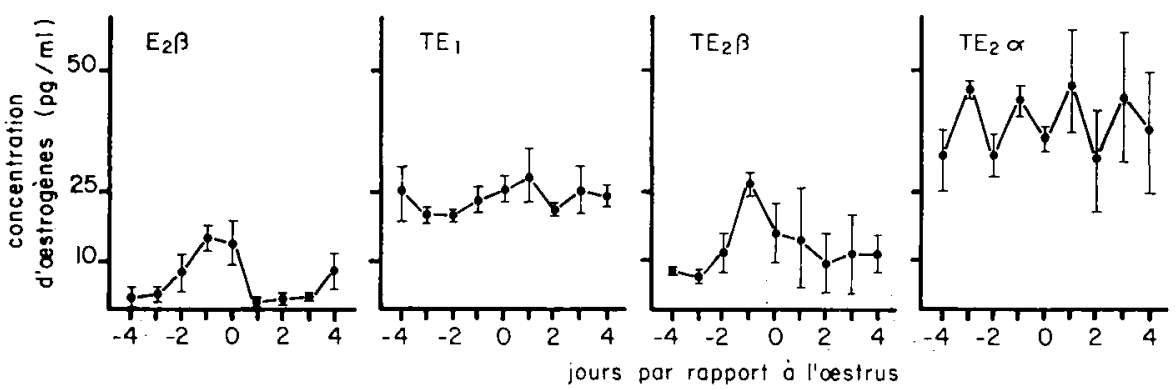

FIG. 2. - Evolution de $E_{2} \beta, T E_{1}, T_{2} \beta$ ef $T_{2} \alpha$ pendant 4 jours avant et après l'oestrus chez trois vaches FFPN. 


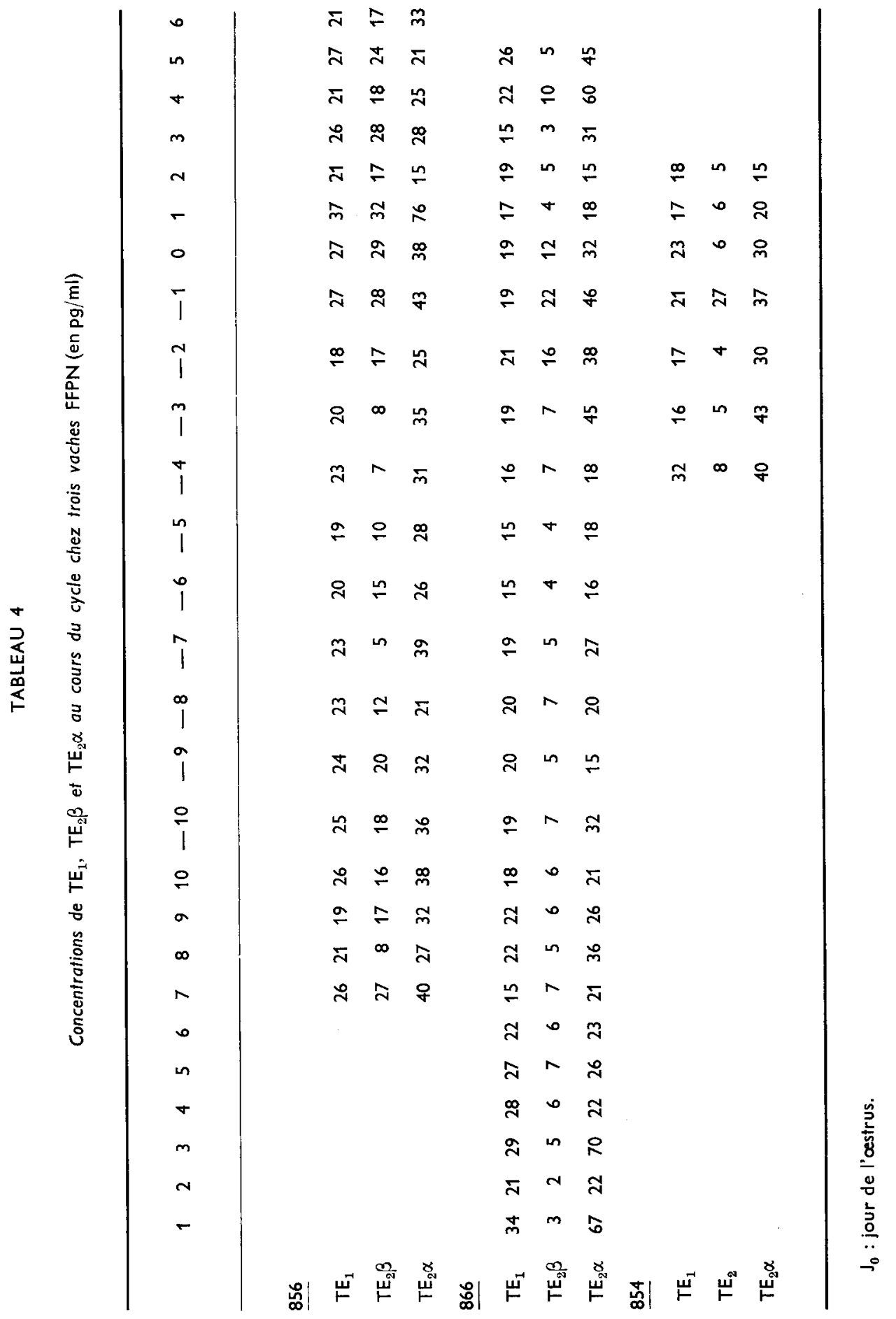


les concentrations les plus faibles étant celles de $\mathrm{TE}_{2} \beta$ ( 2 à $32 \mathrm{pg} / \mathrm{ml}$ ). Les modifications au cours du cycle, en particulier l'augmentation préovulatoire, ne sont nettes que pour $\mathrm{TE}_{2} \beta$. Ce pic dure 3 jours avec un maximum mesuré la veille de l'œstrus, ce qui est tout à fait comparable avec les résultats obtenus sur les mêmes échantillons, pour $E_{2} \beta$. Chez un animal (856) 2 autres périodes d'élévation des concentrations de $\mathrm{TE}_{2}$ ont été observées : vers $J_{7}$ et au milieu du cycle.

\section{Discussion.}

Le dosage que nous proposons permet donc de doser dans le même échantillon $T E_{1}, T E_{2} \beta$ et $T E_{2} \alpha$. Le dosage dans le même échantillon des épimères de $E_{2}$ est rare car leur séparation par chromatographie difficile. L'utilisation de la stéréospécificité de l'oxydation de la fonction $17 \beta-0$ l nous a permis de tourner cette difficulté. Les critères de validité sont satisfaisants, en particulier en ce qui concerne la sensibilité. II faut noter cependant que la précision du dosage de $T_{2} \alpha$ n'est pas très bonne, ceci parce que la mesure radioimmunologique proprement dite est réalisée sur un faible pourcentage de l'échantillon de départ.

Cette technique est relativement longue ce qui en limite l'application à un travail analytique. Une telle étude correspondait à notre préoccupation : trouver une alternative au dosage de l'œstradiol pour suivre la croissance folliculaire. Cette méthodologie pourrait être appliquée à d'autres espèces et à d'autres problèmes physiologiques permettant de sélectionner objectivement, en fonction du buł poursuivi, le stéroïde à doser, stéroïde qui peut être par la suite mesuré par une méthode plus rapide.

Au cours du cycle, les concentrations les plus élevées sont mesurées pour $\mathrm{TE}_{2} \alpha$ puis $T E_{1}$ et $T E_{2} \beta$. Lorsque les valeurs de $T E_{2} \alpha, T E_{2} \beta$ et $T E_{1}$ sont comparées à celles obtenues pour les mêmes stéroïdes non conjugués (Dobson et Dean, 1974), il apparaît que la majorité de $E_{2} \beta$ et $E_{2} \alpha$ est non conjuguée, les différences entre les 2 mesures étant faibles; par contre, les concentrations de $T_{1}$ sont 8 à 10 fois plus importantes que celles de $E_{1}$ non conjugués, ce qui montre que, dans le plasma périphérique, $E_{1}$ circule principalement sous forme conjuguée.

D'un point de vue qualitatif, seul $\mathrm{TE}_{2} \beta$ présente un pic préovulatoire bien défini pic qui est de même importance, en amplitude et en durée à ce qui est obtenu pour $E_{2} \beta$ chez ces mêmes animaux ou lors d'expériences précédentes (Henricks, Dickey ef Hill, 1971 ; Glencross et al., 1973 ; Dobson et Dean, 1974 ; Ectors et al., 1975). L'augmentation d' $E_{2} \beta$ qui est parfois observée vers $\mathrm{J}_{6} \mathrm{~J}_{7}$ et vers $\mathrm{J}_{10^{-} \mathrm{J}_{12}}$ en relation avec une accélération de la croissance folliculaire (Mariana et N'Guyen, 1973) a été aussi observée pour $\mathrm{TE}_{2} \beta$. Contrairement à ce qui a été observé chez la femme (Brown ef Smyth, 1971 ; Loriaux, Ruder ef Lipsett, 1971 ; Hawkins ef Oakey, 1974), l'œstrone conjugué ne présente pas, lors de la période préovulatoire, de variation de concentration plus importante que $E_{2} \beta$. En ce qui concerne $T_{2} \alpha$, il y aurait une tendance pour que les valeurs les plus élevées soient mesurées à proximité de l'œstrus, tendance qui n'est pas évidente sur la courbe moyen ne car les maximums ne sont pas synchrones. Il est aussi possible que ce résultat ait une origine technique : il peut être dû à la mauvaise précision du dosage de $\mathrm{TE}_{2} \alpha$. Ne pouvant pour l'instant améliorer cette caractéristique, afin de juger de l'intérêt de la mesure de $\mathrm{TE}_{2} \alpha$ pour suivre la crois- 
sance folliculaire, ce dosage pourrait être réalisé chez des animaux ayant reçu un traitement de superovulation.

En conclusion, nous proposons une méthode d'analyse qui permet de choisir objectivement en fonction de la situation physiologique le(s) œstrogène(s) qu'il faut doser. L'utilisation de cette méthode pour les échantillons de plasma périphérique recueillis chez la vache au cours du cycle œstral montre que le dosage de $T E_{1}, T_{2} \beta$ ou $\mathrm{TE}_{2} \alpha$ n'apporte aucune amélioration à la qualité des informations obtenues par la mesure d' $E_{2} \beta$ et ne lève aucune des contraintes liées au dosage de cet œstrogène.

Reçu en juillet 1978.

Accepté en mai 1979.

\section{References}

BROWN J. B., SMYTH B. J., 1971. Oestrone sulphate. The major circulating oestrogen in the normal menstrual cycle. J. Reprod. Fert., 24, 142 (abstr.).

CHENAULT J. R., THATCHER W. W., KALRA P. S., ABRAMS R. M., WILCOX C. J., 1975. Transitory changes in plasma progestins, estradiol and luteinizing hormone approaching ovulation in the bovine. J. Dairy Sci., 58, 709-717.

DESSYPRIS A. G., 1975. Testosterone sulphate, its biosynthesis metabolism, measurement functions and properties. J. Steroid Bioch., 6, 1287-1298.

DOBSON H., DEAN P. D. G., 1974. Radioimmunoassay of oestrone, oestradiol-17 $\beta$ and oestradiol-17 $\alpha$ in bovine plasma during the oestrus cycle and last stages of pregnancy. J. Endocr., 61, 479-486.

ECTORS F., BECKERS J. F., BALLMAN P., DERIVAUX J., 1975. Variations du 17ß-oestradiol au cours du cycle oestral chez la vache. C. R. Acad. Sci. Paris, 281, Sér. D, 1257-1260.

GLENCROSS R. G., MUNRO I. B., SENIOR B. E., POPE G. S., 1973. Concentration of oestradiol-17ß, oestrone and progesterone in jugular venous plasma of cows during the oestrous cycle and early pregnancy. Acta endocr., 73, 374-384.

HAWKINS R. A., OAKEY R. E., 1974. Estimation of oestrone sulphate, oestradiol-17ß and oestrone in peripheral plasma : concentrations during the menstrual cycle and in men. J. Endocr., 60, 3-17.

HENRICKS D. M., DICKEY J. F., HILL J. R., 1971. Plasma oestrogen and progesterone levels in cows prior to and during estrus. Endocrinology, 89, 1350-1355.

HENRICKS D. M., HILL J. R., DICKEY J. F., LAMOND D. R., 1973. Plasma hormone levels in beef cows with induced multiple ovulations. J. Reprod. Fert., 35, 225-233.

LEMON M., SAUMANDE J., 1972 . Oestradiol-17 $\beta$ and progesterone after induction of superovulation by PMSG in cattle. J. Reprod. Ferf., 31, 501-502.

LEMON M., SAUMANDE J., 1974. The evolution of ovarian steroid hormones during luteolysis and folliculogenesis in the cow. Europ. J. Obstet. Gynec. Reprod. Biol., 4, S69-S75.

LONGCOPE C., 1972. The metabolism of estrone sulfate in normal males. J. clin. Endocr. Metab. 34, 113-122.

LORIAUX D. L., RUDER H. J., LIPSETT M. B., 1971. The measurement of oestrone sulfate in piasma. Steroids, 18, 463-472.

MARIANA J. C. et N'GUYEN HUY N., 1973. Folliculogenèse chez la vache. Ann. Biol. Anim. Bioch. Biophys., 13, $\mathrm{n}^{\circ}$ hors série, 211-221.

PETERSON A. J., FAIRCLOUGH R. J., PAYNE E., SMITH J. F., 1975. Hormonal changes around bovine luteolysis. Prostaglandins, 10, 675-684.

RUDER H. J., LORIAUX L., LIPSETT M. B., 1972. Estrone sulfate : production rate and metabolism in man. J. clin. Invest., 51, 1020-1033.

SAUMANDE J., 1978. Relationships between ovarian stimulation by PMSG and steroid secretion, 169-194. In SREENAN J. M., Control of reproduction in the cow, Martinus Nijhoff, The Hague/ Boston/London. 
SAUMANDE J., TESTART J., 1974. Plasma levels of estradiol-17 $\beta$ in the prepubertal heifer treated to induce superovulation. Theriogenology, 2, 121-129.

SAUMANDE J., PELLETIER J., 1975. Relationship of plasma levels of estradiol-17 $\beta$ and luteinizing and luteinizing hormone with ovulation rate in superovulated cattle. J. Endocr., 64, 189-190.

TESTART J., KANN G., SAUMANDE J., THIBIER M., 1977. Oestradiol-17 $\beta$, progesterone, FSH and LH in superovulated prepuberal calves. J. Reprod. Fert., 51, 329-336.

THIBIER M., SAUMANDE J., 1975. Oestradiol-17 $\beta$, progesterone and $17 \alpha$-hydroxyprogesterone concentrations in jugular venous plasma in cows prior to and during oestrus. J. Steroid Bioch., 6, 1433-1437. 